\title{
VALIDATION OF OPTICAL-FLOW FOR QUANTIFICATION OF MYOCARDIAL DEFORMATIONS ON SIMULATED RT3D ULTRASOUND
}

\author{
Qi Duan ${ }^{1}$, Elsa Angelini ${ }^{2}$, Shunichi Homma ${ }^{3}$, Andrew Laine ${ }^{1}$ \\ ${ }^{1}$ Department of Biomedical Engineering, Columbia University, New York, NY, USA \\ ${ }^{2}$ Ecole Nationale Supérieure des Télécommunications, GET, CNRS UMR 5141, Paris, France \\ ${ }^{3}$ Department of Medicine, Columbia University, New York, NY, USA
}

\begin{abstract}
Quantitative analysis of cardiac motion is of great clinical interest in assessing ventricular function. Real-time 3-D (RT3D) ultrasound transducers provide valuable fourdimensional information, from which quantitative measures of cardiac function can be extracted. Previously, we presented a method based on four-dimensional optical flow motion estimation for anatomical tracking of myocardium in RT3D ultrasound, from which myocardial displacement fields and dynamic cardiac metrics were computed. In this paper, in order to quantitatively validate our method, we build a truly 3D mathematical phantom of cardiac tissue and blood. Distinguished from previous studies, our work further decomposes tissue impedance into cell kernels and processes all functions in 3D. Instead of simply modeling the myocardium, a "quasi-LV" phantom is built including myocardium and blood. Also all ultrasound probe parameters used in this work are directly estimated from clinical RT3D data instead of using common parameters from 2D transducers. Based on this phantom, simulated RT3D ultrasound data sets are generated for validation to assess the performance of an optical flow based method in tracking myocardial tissues.
\end{abstract}

\section{INTRODUCTION}

Ultrasound is the cardiac screening modality with the highest temporal resolution, but remains limited to twodimensional technology in most hospitals and clinical centers. Development of 3D echocardiography started in the 1990s [1], with real-time 3D (RT3D) ultrasound based on matrix phased arrays. A new generation of RT3D transducers was introduced by Duke University [2,3] and more recently by Philips Medical Systems (Best, The Netherlands) with the SONOS 7500 transducer followed by the iE33 model that acquires a fully sampled cardiac volume within four cardiac cycles. This technical advance increased the spatial resolution and image quality, which makes 3D ultrasound techniques increasingly attractive for routine cardiac clinical diagnosis on cardiac patients. Since RT3D ultrasound acquires volumetric ultrasound sequences with fairly high temporal resolution using a fixed-positioned transducer, it can very well capture cardiac function $[4,5]$ as well as complex $3 \mathrm{D}$ cardiac motion.
In a previous study [6], we developed a framework to derive several dynamic cardiac measures based on fourdimensional optical flow using RT3D ultrasound temporal sequences. This framework was applied to a clinical data set from a heart transplant patient and dynamic measurements agreed with findings in related cardiac biomechanics studies $[7,8]$. In this paper, in order to quantitatively validate our method, we first build a mathematical phantom to mimic acoustic impedance of cardiac tissue and blood. Based on this phantom, simulated real-time four-dimensional ultrasound data sets are generated, using transducer parameters similar to those of the RT3D ultrasound machine from Volumetrics $[2,3]$. Performance of our optical flow based method in tracking myocardial tissues are explored by simulating consecutive RT3D ultrasound frames of the beating heart under controlled motion fields, including translation, rotation, and thickening deformations.

\section{METHODOLOGY}

\subsection{Ultrasound Simulation Methods}

Simulating ultrasonic B-scanning images can be dated back to 1980 [9]. In their paper, Bamber et al. modeled the ultrasonic image formation process in the far field as a convolution of the point spread function (PSF) from the transducer and the scattered distribution of underlining tissues defined by local density and compressibility. Several studies have been performed since then, with more or less elaborate models. In [10], Narayanan et al. used a very simple simulation framework for transmitted pulses and received echo signals to study the influence of the scatter cross-section and the number of scatterers per cells on envelope signal statistics. Their model was derived from the extensive review of first and second order statistics of complex random signals from Wagner et al. [11]. Specific to cardiac applications, a study in [12] demonstrated that the K-distribution is generally more appropriate than the Rayleigh distribution to simulate backscatters from myocardial tissue in the frequency range of 5-15 MHz. On the other hand, Meunier et al. published three papers in 1994 [13] and $1995[14,15]$ to study speckle motion artifacts under tissue dynamics in 2D B-mode images, using a Rayleigh distribution model. The K-distribution is generated by a sparse density of Poisson scatterers (average number of scatterers per 
resolution cell was between 9 and 12), whereas the Rayleigh distribution is generated by a dense distribution of Poisson scatterers (above 12) [12, 16]. A 3D extension of Meunier's model, to generate ultrasound image volumes was proposed in [17] and in [18] for a uniform medium scanned on a rectilinear grid geometry.

In a different context, Jensen [19] developed an extensive ultrasound simulation software package called FIELD in 1996 based on Tupholme-Stepanishen's method, which allowed arbitrary transducer design. For this reason, the current version, FIELD II, is widely used in transducer design.

\subsection{Image Formation Model}

In this work, we used Meunier's simulation framework. Assuming a linear and position-independent point spread function (PSF) of the transducer in the far field, the 3D radio-frequency (RF) echographic signal $R F(x, y, z)$ can be modeled by a $3 \mathrm{D}$ convolution product $(\otimes)$ between the system PSF $H(x, y, z)$ and the impulse response of the tissue $T(x, y, z)[9,14,15]$ :

$$
R F(x, y, z)=H(x, y, z) \otimes T(x, y, z) .
$$

For the situation of a far-field plane-wave that propagates in the $x$-direction in a weakly inhomogeneous continuum, a function describing the tissue echogeneity, $T(x, y, z)$, was derived by Bamber and Dickinson in [9]

$$
T(x, y, z)=\frac{1}{4} \frac{\partial^{2}}{\partial x^{2}}\left(\frac{\rho(x, y, z)}{\rho_{0}}-\frac{\beta(x, y, z)}{\beta_{0}}\right)
$$

where $\rho$ and $\beta$ are the density and compressibility functions deviating from the reference value $\rho_{0}$ and $\beta_{0}$. As shown in [15], the function $T(x, y, z)$ can be reformulated through acoustic impedance $Z(x, y, z)$ leading to :

$$
R F=H \otimes \frac{\partial^{2} Z}{\partial x^{2}}=\frac{\partial^{2} H}{\partial x^{2}} \otimes Z
$$

The final 3D ultrasound image is formed by envelope detection, modeled as an analytical demodulation:

$$
I(x, y, z)=|R F(x, y, z)+j \times \mathbf{H}(R F(x, y, z))|
$$

where $I(x, y, z)$ is the $3 \mathrm{D}$ ultrasound image, $\mathbf{H}()$ is the Hilbert transform that provides the quadrature component of the signal, and $j$ is the square root of -1 .

\subsection{Point Spread Function}

Meunier propose to model the far-field PSF with a cosine function combined with a 3-D Gaussian envelope:

$$
H(x, y, z)=\exp \left(-\frac{1}{2}\left(\frac{x^{2}}{s_{x}^{2}}+\frac{y^{2}}{s_{y}^{2}}+\frac{z^{2}}{s_{z}^{2}}\right)\right) \cos (2 \pi f x)
$$

where $\left(s_{x}, s_{y}, s_{z}\right)$ defines the size of the PSF in axial, lateral, and transverse directions, respectively; and $f$ is the spatial frequency. In most ultrasound systems, $H(x, y, z)$ is a narrowband signal, which allows to discard the second order derivative operator in Equation(3). This assumption is also used to simplify the model in other studies, explicitly in [13] or implicitly in [14, 15, 17, 18].

\subsection{Tissue Model}

The tissue continuum can be modeled as a collection of cells that behave as scatterers. Assuming that at the scale of image resolution, the cells are effectively identical in shape and orientation, a cell kernel function $C(x, y, z)$ is defined at cell location $\left(x_{n}, y_{n}, z_{n}\right)$. We can even assume that the image pixel is large enough so that $C(x, y, z)$ can be approximated by a symmetric function. Thus for each cell, the corresponding acoustic impedance is

$$
Z_{n}(x, y, z)=a_{n} C\left(x-x_{n}, y-y_{n}, z-z_{n}\right) .
$$

And the total acoustic impedance of the tissue for each image pixel $(x, y, z)$ is:

$$
Z(x, y, z)=\sum_{n} Z_{n}=\sum_{n} a_{n} C\left(x-x_{n}, y-y_{n}, z-z_{n}\right) .
$$

This can be decomposed as a convolution product as:

$$
Z(x, y, z)=C(x, y, z) \otimes N(x, y, z),
$$

where:

$$
N(x, y, z)=\sum_{n} a_{n} \delta\left(x-x_{n}, y-y_{n}, z-z_{n}\right)
$$

and $\delta(x, y, z)$ is the Dirac or impulse function. Based on narrowband PSF assumption as in [15], the process function $N(x, y, z)$ can be approximated by the following equation within each image pixel:

$$
N(x, y, z)=\sum_{n=1}^{m} a_{n}
$$

with $m$ is the total number of cells overlaid with one pixel, which can be modeled as a random variable with Poisson distribution. As pointed out in [14, 15], if the beam thickness is large enough to include at least five cells, $N(x, y, z)$ can be modeled as an image with Gaussian histogram whose variance is equal to its mean. Note that under this assumption, the symmetric assumption on $C(x, y, z)$ can be loosened.

Overall, the 3D image formation model that we use is expressed as:

$$
R F(x, y, z)=H(x, y, z) \otimes C(x, y, z) \otimes N(x, y, z) \quad(11)
$$

Compared to previous models used in 3D simulation, as in [17] and [18], this approach further divides the tissue impedance property into a process function and cell kernel functions in order to incorporate tissue properties for the blood and the myocardium.

\subsection{Myocardium and Blood Parameters}

We did not use typical 2D transducer parameters to extrapolate acquisition parameters for $3 \mathrm{D}$ transducer, as done in previous studies. Instead, we use the available parameters from a commercial Volumetrics machine and 
estimate the unknown parameters from the actual RT3D ultrasound data.

\subsubsection{PSF Parameters Setting}

To approximate the actual image resolution of commercial real-time 3D ultrasound, we set the PSF parameters to $(0.5,1,2) \mathrm{mm}$ Full Width Half Maximum (FWHM) in axial, lateral, and transverse directions. The corresponding $\left(s_{x}, s_{y}, s_{z}\right)$ can be derived using the relationship $F W H M \approx 2.35$ std as given in [15]. The transducer frequency is set to $3 \mathrm{MHz}(2.5-3.5 \mathrm{MHz}$ for Volumetrics), as with typical RT3D transducers. The velocity of the acoustic wave in the human body is set to the standard $1540 \mathrm{~m} / \mathrm{s}$ average value.

\subsubsection{Cell Kernels Parameters Setting}

We choose a 3D Gaussian to model the impedance inhomogeneities as in [15]. Thus, the general cell kernel function is expressed as:

$$
C(x, y, z)=\exp \left(-\frac{1}{2}\left(\frac{x^{2}}{\sigma_{x}^{2}}+\frac{y^{2}}{\sigma_{y}^{2}}+\frac{z^{2}}{\sigma_{z}^{2}}\right)\right) .
$$

Similar to [15], we model the elongated myocardial cells with corresponding standard deviations $\left(\sigma_{x}, \sigma_{y}, \sigma_{z}\right)$ $=(10,10,35) \mu \mathrm{m}$ given the fact that myocardial cells are elongated with dimensions around $(20,20,70) \mu \mathrm{m}$. Since myocardial cells are tightly connected and the myocardial fibers are parallel to each other at $100 \mu \mathrm{m}$ scale or even $1 \mathrm{~mm}$ scale, the 1:1:3.5 ratio can be observed at the image pixel scale. Recent studies on 2D or 3D ultrasound simulations only included modeling of the myocardium tissue. Since in actual clinical RT3D echocardiography, both myocardium and blood are imaged, we also build a tissue model for the blood. The average size of the blood cells is about $(10,10,2) \mu m$ [20]. However, since blood cells are not tightly connected to each others and pixel volumes are much larger than individual blood cell volumes, the echogeneity within each blood pixel is mainly determined by the number of cells within each pixel, a random variable with Poisson distribution. If the number of blood cells within each voxel is large enough $(>5)$, the anisotropic property in the cell dimension can be ignored. In this context, the standard deviation can be simply set to the pixel size.

\subsubsection{Echogeneities Parameters Setting}

The final parameter to be determined is the echogeneity for each tissue in order to fully determine the process function $N$. Although it is a very important parameter, previous studies tended to ignore this. None of them reported which value they used or how to determine it. In order to simulate 3D echocardiographic images, we determined this parameter on clinical RT3D ultrasound images. Several clinical RT3D ultrasound volumes were acquired. The myocardium and blood pool were manually segmented, from which average echogeneity values for each tissue was estimated as 100 for myocardium and 30 for blood.

\subsubsection{Imaging Parameters and Tissue Dynamics}

The left ventricle model has a volume size of (150x150x150) and pixel dimension of $(1,1,1) \mathrm{mm}^{3}$, corresponding to $(150 \times 150 \times 150) \mathrm{mm}^{3}$ of tissue volume. Given that the average volume of a myocardium cell is $15,000 \mu^{3}$ [15] and the average volume of blood cell is $90{\mu \mathrm{m}^{3}}^{3}$, there are about 66667 myocardial cells and 11.11 million blood cells per voxel. Considering the extracellular medium, these numbers are reduced to 46667 myocardial cells 4.44 million blood cells per voxel. Since both numbers are much larger than the reference threshold of 5 cells per voxel, the process function $N$ can be safely approximated by a Gaussian distribution.

Regarding the geometry of the heart, a piece of myocardium with geometry similar to a healthy human left ventricle is placed at the center of the image volume and filled with blood. Tissue dynamics including translation, rotation, and thickening are simulated using the same model as in [15] and [18]. Next, a previously developed optical flow (OF) tracking method is applied to track myocardial motion. These results are then compared to the true motion fields of the underlying tissues.

\section{RESULTS AND DISCUSSION}

Acquisition using a 2D matrix-based linear array in Cartesian coordinate system was simulated. Sample crosssection views from the simulated volume are shown in Figure 1.

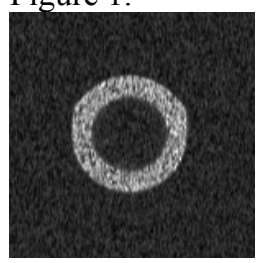

(a)

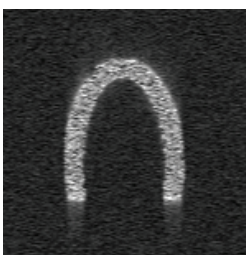

(b)

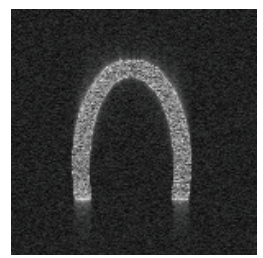

(c)
Figure 1. Sample views from simulated volume: (a) short axis view; (b) elevation long axis-view; and (c) azimuth long-axis view.

Correlation-based 4D OF algorithm is applied to two consecutive simulated RT3D ultrasound volumes.

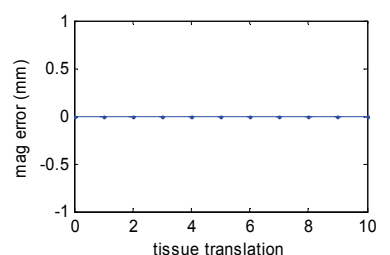

(a)

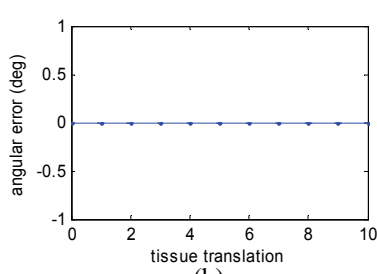

(b)
Figure 2. OF tracking errors during tissue translation: (a) error in magnitude of motion vector; (b) error in angle of motion vector.

The mean and standard deviation for the errors in magnitude and angular displacement during tissue translation are plotted against the displacement magnitude in Figure 2. Similar to the findings in other studies $[15,17]$, errors are minimal. 
The mean and standard deviation for the errors in magnitude and angular displacement during tissue rotation are plotted against the rotation angles in Figure 3. Similar to the findings in other studies $[13,18]$, both the mean and the standard deviation of the errors increase with rotation angles. Fortunately the rotation observed in real clinical RT3D data within two consecutive volumes is usually less than 3 degrees, which is within the interval where our method still exhibits high accuracy.

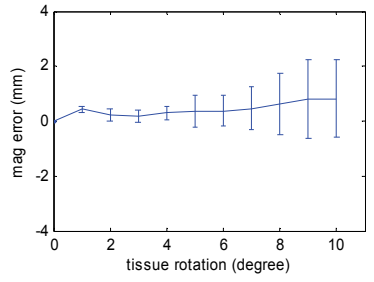

(a)

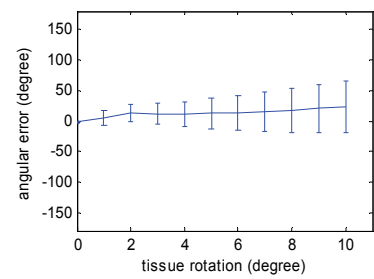

(b)
Figure 3. OF tracking errors during tissue rotation: (a) error in magnitude of motion vector; (b) error in angle of motion vector.

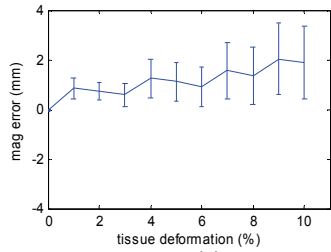

(a)

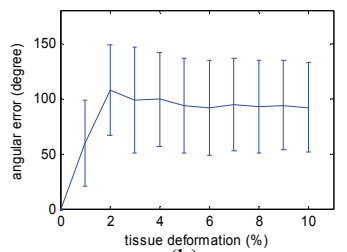

(b)
Figure 4. OF tracking errors during tissue deformation: (a) error in magnitude of motion vector; (b) error in angle of motion vector.

Mean and standard deviation for the errors in magnitude and angular displacement during tissue deformation (thickening or thinning) are plotted against the deformation magnitude (expressed in \%) in Figure 4. The product of deformation percentages in all directions is kept to 1 for the sake of total mass conservation. Similar to the findings in other studies $[14,15,18]$, both the mean and the standard deviation of the errors are increasing with tissue deformations.

\section{CONCLUSION}

A "quasi-LV" 3D mathematical phantom was built modeling both the myocardium and blood. Model parameters were directly estimated from actual clinical RT3D ultrasound machines and data rather than using parameters derived from common 2D transducers. Based on this phantom, simulated RT3D ultrasound data sets were generated to evaluate our previously developed OF tracking software. In future work, real acquisition protocol parameters in RT3D ultrasound and real myocardial motion parameters from a cardiac model [21] will also be incorporated in the model for more realistic simulations.

\section{REFERENCES}

[1] O. T. V. Ramm and S. W. Smith, "Real time volumetric ultrasound imaging system," Journal of Digital Imaging, vol. 3, pp. 261-266, 1990. [2] S. W. Smith, H. G. Pavy, and O. T. Ramm, "High-speed ultrasound volumetric imaging system. I: Transducer design and beam steering," IEEE Transactions on Ultrasound, vol. 38, pp. 100-108, March 1991.

[3] S. W. Smith, H. G. Pavy, and O. T. Ramm, "High-speed ultrasound volumetric imaging system. II: Parallel processing and image display," IEEE Transactions on Ultrasound, vol. 38, pp. 109-115, March 1991.

[4] E. Angelini, A. Laine, S. Takuma, J. Holmes, and S. Homma, "LV volume quantification via spatio-temporal analysis of real-time $3 \mathrm{D}$ echocardiography," IEEE Transactions on Medical Imaging, vol. 20, pp. 457-469, 2001.

[5] E. Angelini, S. Homma, G. Pearson, J. Holmes, and A. Laine, "Segmentation of real-time three-dimensional ultrasound for quantification of ventricular function: a clinical study on right and left ventricles," Ultrasound in Medicine and Biology, vol. 31, pp. 1143-1158, 2005.

[6] Q. Duan, E. Angelini, S. L. Herz, C. M. Ingrassia, O. Gerard, K. D. Costa, J. W. Holmes, S. Homma, and A. Laine, "Dynamic Cardiac Information From Optical Flow Using Four Dimensional Ultrasound," in 27th Annual International Conference IEEE Engineering in Medicine and Biology Society (EMBS), Shanghai, China, 2005.

[7] J. D. Humphrey, Cardiovascular solid mechanics: cells, tissues, and organs. New York, USA: Springer, 2002.

[8] M. B. Buchalter, J. L. Weiss, W. J. Rogers, E. A. Zerhouni, M. L. Weisfeldt, R. Beyar, and E. Shapiro, "Noninvasive Quantification of Left Ventricular Rotational Deformation in Normal Humans Using Magnetic Resonance Imaging Myocardial Tagging," Circulation, vol. 81, pp. 1236-1244, April 1990.

[9] J. C. Bamber and R. J. Dickinson, "Ultrasonic B-scanning: a computer simulation," Phys. Med. Biol., vol. 25, pp. 463-479, 1980.

[10] V. M. Narayanan, P. M. Shankar, and J. M. Reid, "Non-rayleigh statistics of ultrasonic backscattered signals," IEEE Transactions on Ultrasonics, Ferroelectrics and Frequency Control, vol. 41, pp. 845-852, 1994.

[11] R. F. Wagner, M. F. Insana, and D. G. Brown, "Statistical properties of radiofrequency and envelope-detected signals with applications to medical ultrasound," Journal of the Optical Society of America, vol. 4, pp. 910-922, 1987.

[12] L. Clifford, P. Fitzgerald, and D. James, "Non-rayleigh first-order statistics of ultrasonic backscatter from normal myocardium," Ultrasound in Medicine and Biology, vol. 19, pp. 487-495, 1993.

[13] F. Kallel, M. Bertrand, and J. Meunier, "Speckle Motion Artifact under Tissue Rotation," IEEE Transcations on Ultrasonics, Ferroelectrics, and Frequency Control, vol. 41, pp. 105-122, 1994.

[14] J. Meunier and M. Bertrand, "Ultrasonic Texture Motion Analysis: Theory and Simulation," IEEE Transcations on Medical Imaging, vol. 14, pp. 293-300, 1995.

[15] J. Meunier and M. Bertrand, "Echographic Image Mean Gray Level Changes With Tissue Dynamics: A System-Based Model Study," IEEE Transcations on Biomedical Engineering, vol. 42, pp. 403-410, 1995.

[16] T. A. Tuthill, R. H. Sperry, and K. J. Parker, "Deviations from rayleigh statistics in ultrasonic speckle," Ultrasonic Imaging, vol. 10, pp. $81-89,1988$.

[17] J. Meunier, "Tissue Motion Assessment From 3D Echographic Speckle Tracking," Phys. Med. Biol., vol. 43, pp. 1241-1254, 1998.

[18] W. Yu, P. Yan, A. J. Sinusas, K. Thiele, and J. S. Duncan, "Towards pointwise motion tracking in echocardiographic image sequences: Comparing the reliability of different features for speckle tracking," Medical Image Analysis, vol. 10, pp. 495-508, 2006.

[19] J. A. Jensen, "FIELD: A program for Simulating Ultrasound Systems," Medical \& Biological Engineering \& Computing, vol. 34, Supplement.1, Part 1, pp. 351-353, 1996.

[20] D. N. Houchin, J. I. Munn, and B. L. Parnell, "A Method for the Measurement of Red Cell Dimensions and Calculation of Mean Corpuscular Volume and Surface Area," Blood, vol. 13, pp. 1185-1191, 1958.

[21] M. Sermesant, P. Moireau, O. Camara, J. Sainte-Marie, R. Andriantsimiavona, R. Cimrman, D. L. Hill, D. Chapelle, and R. Razavi, "Cardiac function estimation from MRI using a heart model and data assimilation: advances and difficulties," Med Image Anal, vol. 10, pp. 642-56, Aug 2006. 